\title{
Adolescent Organ Transplants: A Lifetime Commitment to Medication
}

\author{
Jihae Jang ${ }^{1}$, Jeannette Dixon ${ }^{1}$, Brittany Franckowiak ${ }^{1}$ and Dr. Cozumel S. Pruette ${ }^{1}$ \\ ${ }^{1}$ Wilde Lake High School, Columbia, Maryland, USA
}

\section{$\underline{\text { ABSTRACT }}$}

Purpose: Medication non-adherence is the most prevalent issue within the adolescent population, where often times adolescent patients will face barriers to achieve adherence. This article sought out to present a narrative review of medication adherence barriers identified and reported in adolescent organ transplant patients, whilst providing potential adherence intervention methods.

Method: A literature review was conducted using the databases PubMed, MasterFILE Premier*, Elsevier, and Google Scholar. The articles were chosen based on their relativity to the topic of medication adherence barriers and interventions in adolescent organ transplant patients. Also, a total of 11 different medical practitioners were contacted for an interview.

Results: Twenty-six studies were chosen to be a part of this review, and based on the content of the studies, five barrier types were developed: "treatment-related barriers", "psychosocial barriers", "transition readiness barriers", and "relationship barriers". The literature review also identified various intervention methods for medication adherence in regard to education, behavior, relationships, and mental health interventions. One interview was conducted with a pediatric nephrologist.

Conclusion: Based on these results it is important to acknowledge that there is not one common barrier among every patient, as each patient develops a unique set of barriers. The review recognized the importance of awareness on the vast possibilities of barriers rather than a generalized approach to adherence.

\section{Introduction}

The World Health Organization defines adherence as "the degree to which the person's behavior corresponds with the agreed recommendations from a health care provider." (World Health Organization [WHO] 2001). Adherence is crucial to a patient who has undergone solid organ transplantation. Solid organ transplantation has become a standard treatment option for various medical conditions and consists of a transplant of the kidney, pancreas, heart, liver, or lungs. Following transplantation, the patients must adhere to a complex treatment regimen. Sustaining strict adherence to the complex regimen is a daily and lifetime responsibility for the patients. The patients must particularly adhere to their immunosuppressant medication to maintain the health of their transplanted organ and their overall survival (Griffin and Elkin 2001). Patients facing non-adherence may ultimately experience various negative post-transplant complications such as additional medical complications, frequent hospitalizations, rejection, allograft loss, and death (Falkenstein et al. 2004; Shaw et al. 2003).

The adolescent population of the transplant patients is at the greatest risk of medication non-adherence, as statistics revealed that non-adherence rates in adolescent patients were "twice as high than younger transplant recipients and almost sixty percent higher than adults aged twenty-four to forty-four" (chop.edu). Due to these high statistics, adolescent patients are at greater risk for negative post-transplant complications, as results also revealed that adolescents, in particular, have the least successful long-term graft survival (Rianthavorn et al, 2004). Adherence can often be difficult to achieve in patients as adolescents often face medication adherence barriers preventing them to achieve complete adherence. These barriers can form from various aspects of a patient's personal mental state, relationships, 
adolescent development, and the treatment regimen itself. The severity and number of barriers faced by each of these patients ultimately affect the level of risk in non-adherence in adolescent patients. Furthermore, the number and types of barriers present in the patient have been revealed to be associated with different measures of non-adherence, such as missed and late doses of medication (Zelikovsky et al. 2008). Given that each adolescent patient has an individual set of barriers, it is essential to specify and address the different types of medication adherence barriers. Also, to address and counter these different barriers, identifying the individual's behaviors provides targets for specific interventions that can be implemented on the patient, allowing both prevention and treatment to adherence barriers. This current article sought out to identify the various different types of medication adherence barriers present in adolescent organ transplant patients and its impact on the adolescent patients' adherence and survival rates. The article also aimed to provide effect adherence interventions to improve the rates of adherence.

\section{Methods}

\section{Search strategy}

A literature review was conducted and articles were collected through PubMed, MasterFILE Premier*, Elsevier, and Google Scholar for studies relating to medication adherence in adolescent organ transplant patients. The articles were chosen based on the relativity to the topic of medication adherence barriers and interventions for adolescents with solid organ transplants. A total of twenty-six articles were selected to be incorporated within this study and were examined to identify common themes.

\section{Interviews}

For this current research, six interview questions were developed, relating to medication adherence barriers, and interventions used to address these barriers. From March 29, 2019, to April 24, 2019, emails containing the interview questions were sent out to eleven different medical professionals from Johns Hopkins Hospital, Stanford Hospital, Harvard University, and Columbia University. There was a total of three responses from medical professionals at Johns Hopkins Hospital. The medical professionals consisted of a cardiologist, a cardiovascular surgeon, and a pediatric nephrologist, who had been forwarded the email by another professional who was initially contacted. The cardiologist and cardiovascular surgeon did not reply to the interview questions and the pediatric nephrologist agreed to an interview over the phone. The interview was conducted over the phone on April 26, 2019, from 11:20 - 11:45 am. With permission from the pediatric nephrologist, the interview was recorded through an iPhone app called, "TapeACall: Call Recorder", to be transcripted and utilized for the review.

\section{Results}

The literature review identified major types of medication adherence barriers that adolescent organ transplant patients experience. After analyzing the different studies on barriers, this review developed five different categories of barriers: "treatment-related barriers", "psychosocial barriers", "transition readiness barriers", and "relationship barriers". The literature review also identified various intervention methods for medication adherence in regards to education, behavior, relationships, and mental health interventions. These barrier types and interventions will be discussed in this review with supportive evidence from literature. 


\section{Treatment-Related Medication Adherence Barriers}

Treatment-related barriers may be related to the physical aspects of the medication such as taste and adverse side effects, and medication complexity and regimen adaptation issues such as medication schedules, planning, and forgetfulness. Medication complexity issues are often due to the quantity and frequency of the patients' prescribed medication. Barriers related to the adolescent patients' treatment ultimately put the patient at greater risk for non-adherence and may increase non-adherent patient behavior.

Adolescent organ transplant patients have reported barriers related to the medication side effects and ingestion issues, although these barriers were not perceived to be a frequent barrier. Parent reports of "my child having a hard time swallowing" and "my child dislikes their medication tastes" had statistically significant associations with higher rejection and death rates (Simons et al. 2010). The significant correlations between the statements and postoperative complications may indicate a relationship between higher rates of non-adherence and ingestion and taste issues. Although ingestion issues are not the most common barrier identified in patients, it may indicate that when these barriers occur, they are particularly important to address. Adolescents also often perceive the cosmetic side effects of their immunosuppressive medication to be of vital importance. Adverse side effects such as hirsutism, gingival hyperplasia, weight gain, and short stature can cause intentional medication non-adherence in adolescents (Griffin \& Elkin, 2001; Lawrence et al. 2008), and adolescent girls may have a potentially higher concern in barriers related to cosmetic side effects such as hair loss. (C. Pruette, personal communication, April 26, 2019). In a study, thirty-five percent of the patients reported feeling uncomfortable with the medication side effects to their appearance and the patients' reports of "don't like what the medication does to appearance" revealed to be correlated with increased late doses (Simon et al. 2010). Although taking medication late is not considered as complete non-adherence, it is still important for the patients to take their medication on time for their transplant health and because an increased number of late doses may eventually lead to complete non-adherence. Along with cosmetic side effects, adolescent patients who have undergone a heart transplant are often prescribed with antihypertensive medications which can cause sexual adverse effects such as impotence. Adverse sexual side effects were perceived to be particularly devastating to male patients (Stuber 2011), which may put male heart transplant patients at a greater risk for medication non-adherence. Patients may intentionally be non-adherent to their medication because they do not fully understand the risks of not taking their medication. They may also decide to be non-adherent and value their sexual reputation and physical appearance over their health due to social stigmas related to beauty standards and sexual performance.

Adolescent organ transplant patients have a highly complex medication regimen that consists of a strict medication schedule. Twenty-eight percent of adolescents patients perceived their medication schedule to get in the way of their daily activities (Hanghøj and Boisen 2014). A common daily activity within patients is their sleep schedule. The medication schedule for adolescent organ transplant patients may be correlated with a greater risk for non-adherence due to the adolescent's regular sleep schedule and morning medication doses. Patients have reported their medication regimen interfering with their sleep schedule (Bullington et al. 2007), and study results revealed eighty-five percent of patients to have missed their morning doses while only fifteen percent of patients missed their evening doses (Simons et al. 2009). The significant difference in medication non-adherence of morning and evening doses may be because adolescents and other individuals may sleep in later on the weekend, and during the week when the adolescent patients are required to go to school, a rushed morning may cause them to forget their medication.

Adolescent organ transplant patients are prescribed numerous medications for their treatment and the number of medications has been identified to be correlated with increased risk for medication non-adherence. Due to the number of their medications, adolescent patients have expressed concerns about having to take their medications too many times a day (Bullington et al. 2007). A study also identified patients' perceptions of having too many pills to have statistically significant correlations with clinical outcomes of death (Simons et al. 2010). The correlation between patient perceptions and clinical outcomes of death indicates an association between the medication quantity and increased rates of non-adherence. The number of immunosuppressant medication prescribed and the overall complexity of the medication regimen requires patients to be organized and responsible for balancing both their personal schedule 
and medication schedule. This balance revealed to be a common struggle within patients as sixty-three percent of adolescent organ transplant patients struggled with barriers related to poor planning and scheduling issues (Simons et al. 2009). Scheduling issues and poor planning may be associated with increased risk of non-adherence because if the patient is away from home or is involved in another activity, it may interfere with their medication regimen and cause them to be unprepared with the medication during the scheduled time. Additionally, barriers relating to scheduling and poor planning may be associated with patient forgetfulness. Forgetfulness is one of the most frequently occurring medication barrier identified in medical studies and older adolescents may often be more vulnerable to forgetting their medication possibly due to the decrease in parental supervision (C. Pruette, personal communication, April 26, 2019). Patient reports revealed that twenty-eight percent of adolescent organ transplant patients regularly forget to take their medication (Wray et al. 2006), and forgetfulness also seemed to affect patients, whether they were intentionally or non-intentionally non-adherent (Bullington et al. 2007). Adolescent organ transplant patients may also forget their medication because of the preoccupation of other activities which corresponds to scheduling and planning issues or may simply not remember to take their medication.

In regard to both scheduling and planning related barriers and barriers related to forgetfulness, patient organization and responsibility plays a large role in the presence of these barriers. During the adolescent age, adolescents develop their executive functioning skills which consist of their cognitive skills. Compared to healthy adolescents, adolescent organ transplant patients revealed to have significantly greater executive dysfunction and thirty-two percent of adolescent organ transplant patients were identified to have significant deficits in meta-cognitive abilities which consists of the patient's' ability to plan and organize (Gutierrez et al. 2016). Forgetfulness, poor planning, and scheduling related barriers may significantly affect medication adherence in these adolescent patients because of their executive dysfunction. The dysfunction in the patients' meta-cognitive abilities may cause the adolescent to struggle with more medication complexity barriers, ultimately leading to greater non-adherence.

\section{Psychosocial Medication Adherence Barriers}

Psychosocial barriers have been reported to be present in many adolescent organ transplant patients. Mental health issues, such as depression, anxiety, and post-traumatic stress symptoms have been most frequently reported in these adolescent patients. During adolescence, patients often have concerns about fitting in and being in control, and playing into both mental health and adolescent issues, the patients' perception and mindset have revealed to affect the overall patient effort in treatment.

Adolescent organ transplant patients who face mental health issues have shown to be more vulnerable to medication non-adherence. Studies have revealed that depression, anxiety, and post-traumatic stress disorders are commonly present in these patients. Mental health may be a major issue in these patients because, during adolescence, the patients may have increased stress as they must manage both their transplant health and additional responsibilities such as school. The increase in responsibilities and stress may increase a patient's risk for anxiety and depression. A study discovered that the severity of mental illnesses, such as anxiety, depression, and post-traumatic stress disorder, had a significant correlation with the total number of adherence barriers and the amount of miss medication doses (McCormick et al. 2013). In correlation, a strong association between non-adherence and psychosocial barriers such as depression, anxiety, and inadequate social support was identified (Fredericks et al. 2011). Mental illnesses may often develop if a patient lacks social and emotional support. During adolescence, a strong support system is vital to maintaining a healthy mindset as adolescent patients will need guidance to independence and may experience a series of mental and physical changes with their body. Mental health issues may cause an increased risk of medication nonadherence because illnesses such as depression may cause a lack of motivation within a patient, and if the patient exhibits depressed behavior such as social withdrawal, it may prevent them from utilizing their support system for help in their treatment. Because a support system is necessary within maintaining both a healthy mental state and healthy organ transplant life, patients with mental illnesses and inadequate social support will ultimately be at an increased risk for medication barriers and overall non-adherence. 
Often times, adolescent organ transplant patients who develop mental illnesses display a lack of effort in their treatment regimen. Mental illnesses such as depression may cause a patient to develop a negative and non-motivated attitude and mindset. Particularly in these adolescent patients, thirty-three percent have expressed feeling tired of living with their illness and tired of taking their medication (Simons et al. 2010), indicating the development of negative perceptions to their treatment. A lack of motivation is a common symptom in depression, which affects an individual's behavior and mindset. Depression may cause the patient to exhibit non-adherent behavior and a lack of motivation to maintain treatment responsibilities such as taking their medication. The behaviors affected by depression and a lack of motivation may eventually lead to non-adherent behavior and increase the patient's risk for death and rejection. Patients have also expressed being angry or depressed toward their illness (Bullington et al. 2007). This feeling of anger or depression may be due to the frustration of all the responsibilities they must maintain for the remainder of their life. Being unable to be fully healthy like their healthy peers despite adhering to treatment, may cause patients to be frustrated towards their illness and act on non-adherent behavior.

In adolescent organ transplantation, the organ transplant itself has revealed to be a large psychiatric burden on patients. Patients have shown to develop symptoms of post-traumatic stress disorder. A study revealed that posttraumatic stress symptoms were present in forty-three percent of the patients while a relatively high rate of depression was also revealed in the patients (Evan et al. 2014). Patients who have post-traumatic stress symptoms may be more prone to other mental illnesses such as depression and anxiety. Possessing symptoms of post-traumatic stress disorder may also cause an increase in the struggle for the adolescent patient to cope with their mental health. This may overall cause an increase in risk for medication non-adherence as the patient may struggle to maintain the responsibilities of their organ transplant alongside their mental health issues.

Adolescence is often a time of identity development and independence. During their development, adolescent patients often exhibit risk-taking behaviors and a desire for normalcy and freedom. Adolescent organ transplant patients have reported deliberate non-adherence to their medication regimen in attempts to appear normal. Patients have reported concerns about feeling different and taking their medication in front of their peers (Simons et al. 2010; Hanghøj and Boisen 2014). Correlating to the patients' concerns, patients who scored higher on disease frustration/adolescent issues, which consisted of "being tired of taking medications" and "not wanting friends to notice", on the Adolescent Medication Barrier Scale (AMBS), revealed to have increased late medication doses (McCormick et al. 2013). Supporting McCormick's findings, patient reports of not wanting to be seen taking medication had statistically strong associations with increased rejection episodes, indicating increased non-adherence (Simons et al. 2010). Adolescent patients may be deliberately non-adherent in attempts to feel normal and not be seen taking medications. Taking immunosuppressive medication in front of their healthy peers may cause the patient to feel different and not fit in, causing them to act on their desire for normalcy.

Adolescent organ transplant patients have been restrained and hospitalized for an extended period of time, and are responsible for a treatment regimen throughout their life. Patients who have gone through life-endangering organ diseases and have been discharged recently after being restricted in a hospital may find a sense of freedom and invincibility (Kirklin, 2015). Being newly introduced to feeling healthy may cause the adolescent to act on risk-taking behaviors such as not taking their medication. These adolescent patients may be unable to realize that they are still sick with a chronic illness despite not being at a life or death situation. Also, because adolescents have not fully developed their risk assessment skills, impulse control, or organizational abilities (Taddeo et al. 2008), they may be at greater risk for risk-taking behavior which may affect their risk for overall non-adherence. The lives of these patients are heavily affected by their organ transplant due to their medication regimen, doctor appointments, and overall treatment. In general, chronically ill adolescent patients have reported feeling that their disease controlled their lives (Hanghøj and Boisen 2014). Adolescent organ transplants patients may also feel a lack of control in their lives due to their illness. This may cause them to act on non-adherent behaviors such as missing medication doses, in attempts to gain control of their lives. 


\section{Transition Readiness Medication Adherence Barriers}

The transitional period from pediatric care to adult care for an adolescent organ transplant patient is a vulnerable time for medication non-adherence due to the increase in treatment responsibilities and new health care team. The adolescent patient must become more independent in their treatment responsibilities, and the transfer of responsibilities may cause the patient to be at greater risk for medication non-adherence if the patient is not well prepared and developed for autonomy. The patient and family also may face adherence barriers as they must adjust to the new adult care providers and the environment.

Patients must adopt more treatment responsibilities during the time of treatment transition, and the shift in responsibilities may cause an increase in risk for medication non-adherence. A study identified that overall transition readiness was correlated with increased adolescent involvement in treatment and decreased transition readiness was correlated with increased parent involvement (Gilleland et al. 2012). This correlation may indicate that patients who were responsible for their own treatment during pediatric care were more prepared for transition while patients who depended on their parents were less prepared due to the lack of experience in taking charge of their treatment. Also, patient reports of avoiding responsibilities due to reasons related to anxiety/fear about being more responsible, comfort with parents handling their daily tasks, and not trusting themselves with their medication (Lochridge 2013). The patient's concerns may stem from the fact that many of these patients have been dependent on their providers and parents throughout their time as a patient. Patient avoidance of responsibilities may be because of the lack of exposure to acting independently and patients may develop anxiety as the thought of undertaking new responsibilities may seem overwhelming. Additionally, a statistically significant correlation was revealed between patient reports of "sometimes I don't realize when I run out of pills" and the amount of missed medication doses (Simons et al. 2010). While in contrast, adolescent reports of filling up their own medication were correlated with greater transition readiness and treatment understanding (Gilleland et al. 2013). This indicates that adolescents who have more medication responsibilities before transition are more prepared to take on the responsibilities for adult care, while patients who are dependent on their parents to take on responsibilities such as refilling their medication, may have a harder time adjusting to the increased autonomy and self-management in adult care.

Adolescent patients who have demonstrated a greater understanding of their treatment and illness often displayed greater medication adherence and treatment transition readiness. In a study, the majority of the patients revealed basic knowledge of their illness but only a few displayed a complete understanding of their transition to adult care. Patients who demonstrated more understanding of their illness revealed to be better prepared for the transition and were more likely to directly communicate with their physician (Clarizia et al. 2009). In relation, a significant correlation was discovered between overall transition readiness, patient knowledge, and patient reports of calling in for medication refills (Gilleland et al. 2013). Adolescent patients who had less understanding of their illness and treatment may not understand the critical importance of medication adherence, the risks to non-adherence, and their overall treatment. This lack of understanding may decrease the possibility for the adolescent patient to be capable of executing treatment responsibilities. Additionally, the lack of direct communication between the patient and provider may indicate weak doctor-patient relationships, decreasing a patient's outlet of treatment information. The significant correlation between patient knowledge and treatment transition may be because those patients who were able to directly talk to their physicians were able to ask more questions and report any problems that needed to be addressed. The patient's increased knowledge in their treatment would allow them to know what medication they needed and when and where to refill their medication individually, without the help of their parents.

In regard to both treatment responsibility and transition readiness, the adolescent's executive functioning has been significantly correlated with the patient's performance in these areas. In a study, twenty-five percent of patients exhibited significant executive dysfunction and within those twenty-five percent, thirty-two percent of them had significant dysfunction in metacognition which consists of a patient's ability to plan, organize, and manage their treatment (Gutierrez et al. 2016). The high levels of metacognitive dysfunction among transplant recipients may cause the patient to struggle with medication barriers related to forgetfulness, poor planning, and scheduling, overall increasing their 
risk for medication non-adherence. The metacognitive dysfunction may significantly impact an adolescent's capability to autonomously engage in behaviors required to follow a complex medication regimen. It may also cause the adolescent to struggle with handling increased responsibilities without the support of their parents, causing them to be unprepared for treatment transition, and at greater risk for medication non-adherence.

In adolescent organ transplant treatment, parents often struggle with their child's treatment, affecting the adolescent's treatment transition. Often during treatment transitioning, confusion can arise among the patient and family in regard to who is responsible for the medication regimen (Shellmer et al. 2011). Additionally, there revealed to be an increase in tension between parents who desired to maintain control over their child's treatment and an adolescent's autonomy regarding treatment transitioning (Kim and White 2017). Correlating to both studies, parents have also displayed having a hard time entrusting treatment responsibility to their child. (Hanghøj and Boisen 2014). Particularly in patients who were transplanted as a young child, parents have traditionally taken responsibility for their child's treatment to ensure that their child stays alive. Throughout the many years of responsibilities, it is understandable that the parent may struggle to release their control over their child's treatment and trust their child to make independent decisions regarding their care. The parent's refusal to delegate responsibilities may interfere with the transfer of decision-making responsibilities from parents to adolescents and hinder the adolescent's aim for autonomy regarding their medical regimen. Additionally, the new shift in responsibilities may create confusion in regard to who is responsible and cause the patient to be less prepared to transition to adult care facilities as both the parent and patient are still adjusting to the new responsibilities.

During the transitional period, there may also be negative connotations attached to adult care facilities and cause adolescent patients and parents to develop negative perceptions of adult care. In a study, patient interviews revealed negative viewpoints of adult care facilities, such as getting lost, sensing that the staff was unfriendly, long lines, the unpleasant appearance of the facility, feeling too young in comparison to everyone else, and not being able to schedule multiple appointments within the same visiting. Correlating to the patient's perspective, the parents also reported concerns regarding feeling abandoned by their pediatric care providers and concerns on the adult care provider's competency (Lochridge et al. 2013). Trust is a vital factor in maintaining a stable health care team with the patient, and providers who preserved confidentiality and displayed more competency were shown to be more trusted by adolescent patients (Fredericks et al. 2011). Because the patient and family would have new adult care providers, the trust would have to be rebuilt between the patients, parents, and adult care providers. Without the previously built trust between the provider and patients, there may be an increased risk in medication non-adherence as there will be a lack of trust and communication, creating a barrier for the provider to be able to access and address the present issues in the patient. Additionally, discrepancies have been identified in parents regarding when the transition would take place, and if the adolescent had to transition at all (Lochridge et al. 2013). The discrepancies may increase confusion in regards to the patients' transition and take away the patient's opportunity for an organized and controlled period of time to prepare their treatment transition responsibilities. The period of discrepancy and ambiguity may cause an increased risk for medication non-adherence in the adolescent patient.

\section{Relationship Medication Adherence Barriers}

Adolescent organ transplant patients must have a support system in place throughout their organ transplantation treatment. Adolescent patients may face barriers related to their relationship with their parents, peers, or health care provider. The barriers may stem from a lack of support and the ability to delegate responsibilities with their parents or may associate with the patient's desire for normalcy within their peers. Patients may also face barriers in regards to doctor-patient interaction and trust. Whomever the adolescent may face barriers with, an unstable relationship with any of these people will affect the strength of the patient's support system and will ultimately increase the risk of medication non-adherence and post-operative complications.

Adolescent relationship with their peers has been shown to affect adolescent organ transplant patients' risk for non-adherence. Adolescent patients reports of "I do not want other people to notice me taking the medicine" had 
a statistically significant association with increased rejection rates (Simons et al. 2010). The significant correlations between the statements and negative post-operative complications may indicate a correlation between higher rates of non-adherence and unstable patient-peer relationships. Adolescents also expressed fear that their peers would learn about their disease and concerns about feeling different from their peers (Taylor et al. 2010). These patient reports correlate with psychosocial medication adherence barriers related to the adolescent's desire for normalcy and social acceptance, where the adolescents often revealed concerns of fitting in and appearing normal in front of their peers. A patient's medication regimen requires the patient to take medication multiple times throughout the day, causing the action of taking medication at school and in front of their peers to likely occur. Patient reports of not wanting people to see them taking their medication may have a correlation with their concerns about appearing different and their peers learning about their disease. Patients may intentionally not adhere to their medication regimen to avoid taking their medication in front of their peers and to prevent their peers from finding out about their illness, which will affect their ability to appear normal. The patients may feel like their illness has isolated and made them stand out in front of their peers. However, in contrast to these statements, another study revealed that some adolescents feared rejection from their peers if they were not sick anymore due to the creation of a special identity through their disease (Bullington et al. 2007). This fear of rejection may be due to the fact that many of the adolescent patients who were transplanted as young children have lived with their disease for most of their life, causing it to develop into a part of their identity. Adolescent patients and their relationship with their parents have often been shown to affect the adolescent's medication adherence. A study revealed that impaired family function was associated with higher rates of nonadherence (Shellmer et al., 2011), where family stress and bad home life listed as indicators of a conflictive environment (Johnson et al 2008). The impaired family function may create an unstable environment for the patient, causing it to be more difficult for the patient to receive the support that is necessary for treatment and medication adherence. Additionally, a weak relationship with their parents may cause the adolescent to struggle with communicating any issue or problems they need support with, which may relate to how some patients were afraid to ask their parents for help with their medication (Bullington et al. 2007).

The parents of the adolescent patient may also affect the adolescent's ability to adhere to medication in relation to treatment responsibilities. Adolescents must eventually learn to take on all the responsibilities of their treatment and transition to adult care. In relationship to transition-related medication adherence barriers, parents of the patients have reported struggling with "letting go" and delegating treatment responsibilities with the adolescent (Hanghøj and Boisen 2014). The parents' difficulty in delegating treatment responsibilities may cause the adolescent to become too dependent on their parents and prevent them from developing independence. This may also affect the patient when it is time to transition, as they are less prepared to take on the treatment responsibilities, ultimately increasing their risk for medication non-adherence.

Adolescent organ transplant patients with a poor relationship and a lack of trust with their doctors are at a greater risk for non-adherence. A study revealed that a lack of support from health care providers was associated with poor communication with the doctors. (Taylor et al. 2010) Poor communication may indicate a lack of information from doctors which may be caused by a lack of direct patient involvement. A lack of patient involvement may cause the adolescent to lose trust in the doctor and feel left out in treatment, especially in a case where they feel like they are not being told all the information told to their parents. This can cause discrepancy and a lack of trust between the patient and the doctor, creating a greater risk for medication non-adherence. Also, a weak relationship between the doctor and patient may cause a lack of communication which can lead the patient to not inform their doctor about present issues and conflicts that may affect the patient's non-adherence. This may prevent the problem from getting addressed and cause continuous non-adherent behavior. Additionally, in a study with both healthy teens and teens with chronic illnesses, adolescents expressed fear of being judged by the doctor. (Britto et al. 2004). The fear of being judged may also cause the adolescent patient to withhold important information that could be drastic to one's health or affect the patient's adherence. 


\section{Interventions to Medication Non-adherence}

A majority of adolescent organ transplant patients will face medication barriers at one point in their treatment and many will face a risk of medication non-adherence. Despite the presence of non-adherence, there are various interventions being currently implemented to improve medication adherence in adolescent patients. These interventions address non-adherent behaviors, lack of education, unstable relationships, mental health-related barriers, and promote self-management. Incorporating different aspects of these interventions into an adolescent patient's treatment may decrease their risk for medication non-adherence and address possible adherence barriers.

Due to the current technology-based period, many studies have implemented the use of technology (i.e. Texting, phone apps.) to increase medication adherence. In a study, an intervention method was implemented where researchers separated the patients into the intervention group or the control group. Patients in the intervention group weekly logged onto a provided website to receive materials on disease management, communication skills, and lifestyle tips. The patients were also sent three to five text messages a week to ensure completion and understanding. Text messaging also acted as a communication portal with the healthcare team. Participants in the intervention group demonstrated an increase in disease self-management and health-related self-efficiency. Additionally, the number of patient-initiated communication increased between the patient and their health care team. The study resulted in a total of seventy-eight percent adherence in the intervention group participants indicating a successful intervention method. (Huang et al. 2014). In another intervention study utilizing text messages, the patients were sent text message medication reminders for medication intake. If the patient did not respond within a certain time frame, an additional text message was sent out to the caregiver. The text messages were constructed as "Take [name of medication] at [set time]. To confirm intake press REPLY, type CARE 1, and press SEND.” Organ rejection episodes decreased from twelve cases to two and patient standard deviation scores of patient tacrolimus levels decreased indicating improved medication adherence (Miloh et al. 2009). In both of the intervention studies, text message reminders revealed to be an effective method to address medication adherence barrier in adolescent patients. Barriers related to forgetfulness seemed to be particularly successfully addressed when utilizing text messages. Additionally, the use of electronics and the internet revealed to successfully provide informational support to the patients, allowing them a greater understanding of their illness, along with greater independence in regards to communication and disease management. The study by Miloh et al. was also able to successfully delegate treatment responsibilities between the patient and their caregiver, allowing the patient to take most of the responsibility and act autonomously while continuing to provide parental supervision when needed.

Behavioral interventions were also shown to be effective in addressing medication adherence barriers. One specific study utilized intervention visits to provide adherence intervention methods to patients. During the intervention visits, the patient, and a coach created an Adherence Support Team where patients could choose whether to include their caregivers or not. While forming the Adherence Support Team, the patient and coach developed responsibilities for each team member regarding medication adherence. The coach educated the adolescent on their immunosuppressive medication through slide presentations identified adherence barriers and used "Action-Focused Problem Solving" to address the most important patient chosen barriers. The "Action-Focused Problem Solving" method consisted of brainstorming viable solutions to barriers, assessing the potential solutions, and selecting one solution to implement. The participants could also decide to receive text messages, emails, or visual cues to remind them to take their medication. (Foster et al. 2018). During the intervention interval, medication adherence was $100 \%$ on seventyeight percent of the days and on time adherence, where medication was taken on time, patients were adherent $100 \%$ on seventy-three percent of days. Foster et al.'s study also utilized text messages as an intervention but also provided supplemental behavioral support and interventions to address present adherence barriers. The interventional visits may significantly help increase medication adherence in patients who are facing or are at risk for medication adherence barriers by helping them be able to independently address these barriers. This intervention also successfully engaged the patient into the treatment making decisions through a patient-driven method rather than one controlled by the 
health care provider or parent. This study may have shown successful results due to its success in developing a strong doctor-patient relationship, by developing a partnership with the patient in regards to the treatment team.

Psychological interventions have been shown to improve treatment motivation which has been linked to improved medication adherence. Patients who have undergone an organ transplant are provided a psychiatrist on their health care team, yet therapy alone may not be enough to address every barrier faced by the patients. A study described an intervention called Motivational Interviewing which is a personalized, patient-centered approach to health behavior change that helps resolve doubt, improves motivation for change, and facilitates patient decision-making. It promotes personal control and autonomous decision-making, focusing on the individual's goals and values. (Fredericks et al 2011). This method may be helpful in adolescent organ transplant patients who face psychosocial related barriers related to a lack of commitment and motivation to treatment and recovery. Additionally, Motivational Interviewing may be particularly helpful during the time of treatment transition where adolescent patients must adopt more treatment responsibilities and decision-making behaviors.

Additional treatment education and behavioral interventions may also consist of simple behaviors or changes. Studies have also found that linking medication intake with daily activities such as brushing teeth, to be helpful in remembering to take medication. Visual reminders such as leaving medication where it is noticed have also been recommended to prevent forgetfulness related barriers (Wray et al. 2006) Also, in regards to education, a study recommended verbal discussions with health care providers, written material, and videos as possible educational interventions (Opiola and Steuer 2017). An effective educational method called "Teach Back" was described where the physician would assess the patient's treatment knowledge by asking the patient to explain their understanding in their own words after they were given the educational material. It was also stated that developing a partnership with the patient and engaging the patient in the education and treatment planning would allow the greatest chance of transplant success. (C. Pruette, personal communication, April 26, 2019). Actively engaging and involving the patient in the doctor appointments during educational and treatment discussions is vital to the overall success in a patient because this may improve the patient's genuine understanding of their illness and the dangers of non-adherence. Increasing the patient's treatment knowledge may ultimately improve the patient's adherence rate and treatment transition readiness, as treatment understanding is a vital factor for a patient's transition to adult care. In addition to patient education, family-centered educational program with videos and written material, to be reviewed at home as a family, were revealed to significantly increase treatment understanding. (Lawrence et al., 2011). Along with increased patient treatment understanding, family-centered interventions may also help provide a stable support system as the parent and caregivers may be able to better understand the adolescent patients' feelings and issues that may affect their adherence. Also, during doctor visits and educational interventions utilizing language that the patient and parent can understand has been noted to be very important when delivering educational or medical information (Taddeo et al 2008). Avoiding "medical talk" and complicated vocabulary will allow the patient and family to better understand the information being given.

Doctor-patient interaction has been revealed to be heavily correlated with the stability of doctor-patient relationships and its effect on medication adherence. A study revealed adolescents to highly value confidentiality in regards to what is told to their parent and what is told to them. (Klostermann et al. 2005). Adolescent patients may not be aware of a physicians' obligation to inform or to not inform parents about private information, therefore, it may help patients to be informed of the doctors' obligation to confidentiality. Additionally, in another study, patients rated the different factors of a good doctor-patient relationship, where doctor trust and respect, patient power and control in treatment, and caringness and closeness with the physician were revealed to be the top three in importance to adolescent patients (Britto et al. 2004). Doctor trust and respect may be established by including the adolescent in all treatment decisions and informing them of any complications or other medical information such as medication side effects. Failing to inform patients of all possible medication side effects may cause the adolescent to lose trust in their doctor if they begin to experience uninformed adverse side effects. In regards to a patients' feeling of power and control in treatment, it may be effective to increase patient involvement and to talk directly to the patient rather than through their parent. This feeling may also be of high importance as adolescent patients often exhibit non-adherent behavior 
to gain the feeling of control of their life (Hanghøj and Boisen 2014). Increasing patient involvement in treatment has also been shown to help prepare adolescents regarding responsibility and transition. Additionally, parents have reported that implementing procedures executed in adult care such as having the parent step out during clinic visits, having the patient co-sign forms of consents, or directly asking the patient about medications, were effective actions to help transition preparation (Lochridge et al. 2013). Building trust between the patient and the health care provider is vital to adherence, as the provider must be able to successfully interact with the patient, and identify and address any health issues or medication barriers present in the patient's life. Having a supported level of trust may ultimately provide the patient with a significantly increased chance of transplant success.

Adolescent organ transplant patients, whether they face medication adherence barriers or not, can benefit from the various intervention methods. The intervention methods can address present adherence barriers and prevent future barriers in the patients. Implementing different aspects of these intervention methods to address the specific medication adherence issues in an individual patient may allow the adolescent patient the highest possibility of successful treatment. It may also ultimately allow the greatest chance of survival and adherence, along with a decrease in risk for post-operative complications.

\section{Discussion}

Medication non-adherence in adolescent organ transplant patients continue to be of high prevalence, ultimately putting the adolescent patient at high risk for detrimental post-operative complications such as rejection and death. The high rates of non-adherence are heavily dependent on the severity and quantity of medication adherence barriers faced by these patients. These barriers were categorized into four barriers in this study: treatment-related barriers, psychosocial barriers, transition readiness barriers, and relationship barriers. Treatment-related barriers were commonly related to the complexity of the medication regimen which was profoundly affected by the adolescent patient's executive functioning, and the adverse aspects of the immunosuppressant medication. The patient's perception of the adverse medication side effects correlated with the psychosocial barrier for the desire for normalcy, in relation to issues on adolescent development. Post-traumatic stress symptoms, depression, and anxiety also revealed to be present in many adolescents, often being exhibited through a lack of treatment commitment. In relation to the treatment-related barriers, during the transition from pediatric care to adult care, patients were particularly at risk for non-adherence due to barriers associated with increased responsibilities and relationship barriers with parents and new adult health care providers.

After analyzing the medication adherence barriers, this study discovered that various categories and specific adherence barriers were often associated with each other. Due to the association between the various barriers, by identifying one of the major barriers to non-adherence in the adolescent patient, health care providers may ultimately be able to address additional adherence barriers the patient was struggling with. Furthermore, identifying the specific barriers present in these patients would allow there to be specific targets when implementing intervention methods. This overall allows specific barriers to be addressed in the patient rather than generalizing the non-adherence issues and barriers. This study concluded that there is ultimately no set barrier or most common barrier within patients as each patient's situation varies on the patient themselves. It is important to note that each individual patient develops a unique set of barriers and severity of non-adherence. If the barriers faced by an individual are accurately measured, health care providers would also be able to better understand and identify the severity of non-adherence and distinguish whether the non-adherence was intentional or unintentional. This indicates the high importance of a stable doctorpatient interpersonal relationship as the health care providers are ultimately the ones who are the ones who provide medical support for the barriers. By establishing stable and strong interpersonal relationships between doctors and patients, a more accurate and detailed measurement of the patients' current unique barriers. By evaluating all of these aspects and understanding what barriers must be addressed, the most effective intervention can be implemented on the patient by incorporating different aspects of different intervention methods to fit the patient's specific needs. 
Ultimately by addressing the greatest number of barriers in the patient, the overall risk and level of non-adherence in the patient will decrease and allow the greatest chance of adherence and survival for the patient.

There are many limitations to this study as this researcher was given a limited time to conduct overall the literature review. There were also limitations on the overall primary data collected as this research was only able to conduct one interview with a pediatric nephrologist. This provided a limited perspective on medication adherence in specific organ transplant patients, and it is important to recognize that the information collected from the interview may differ in adolescent patients who have had an organ transplant different from kidney transplants such as heart and liver. It is also important to recognize that this review does not cover all the medication adherence barriers present in patients, as each patient has their own set of unique barriers. It is also necessary to acknowledge that although barriers related to financial issues were not mentioned in this review, they are active, potential barriers to these patients. Additionally, the categories of medication adherence barriers are not permanently set and may vary from different studies, as in this study they were based on the qualitative data collected in the literature review.

\section{Conclusion}

There is a vast variety of medication adherence barriers within adolescent organ transplant patients, and it is important to recognize and be aware of these specific possibilities of barriers rather than having a generalized approach to adherence. Although some studies may indicate a higher frequency of barriers related to forgetfulness, organization issues, and social aspects, there ultimately is not a set barrier that is most common among patients. Each individual patient will develop their own unique individualized set of barriers, depending on their personal situation. Therefore, it is essential for health care providers to accurately identify and address the unique barriers within a patient, allowing the greatest chance of adherence and decreasing the risk of post-operative complications.

\section{Limitations}

There are many limitations to this review paper as there was a one-year time restraint to gather data and may lack additional factors such as financial factors. Additionally, it is important to acknowledge that the information and conclusion discovered from this review may not apply to all patients as every patient is different and reacts uniquely to treatment approaches.

\section{Acknowledgments}

I would like to thank my Wilde Lake High School teachers: Ms. Dixon, for guiding me throughout the entire process of this research and Ms. Franckowiak, for helping me advance my review article. I would also like to thank Dr. Cozumel S. Pruette for enhancing my understanding on this research topic. This study has not received any funding, and the author and contributors do not have any conflict of interest.

\section{References}

Britto, M. T., DeVellis, R. F., Hornung, R. W., DeFriese, G. H., Atherton, H. D., \& Slap, G. B. (2004). Health care preferences and priorities of adolescents with chronic illnesses. Pediatrics, 114(5), 1272-1280.

Bullington, P., Pawola, L., Valenta, A., Briars, L., \& John, E. (2007). Identification of medication non-adherence factors in adolescent transplant patients: The patient's viewpoint. Pediatric Transplantation, 11(8), 914-921. 
Clarizia, N. A., Chahal, N., Manlhiot, C., Kilburn, J., Redington, A. N., \& McCrindle, B. W. (2009). Transition to adult health care for adolescents and young adults with congenital heart disease: Perspectives of the patient, parent, and health care provider. The Canadian Journal of Cardiology, 25(9), 317-322.

Evan, E. E., Patel, P. A., Amegatcher, A., \& Halnon, N. (2014). Post-traumatic stress symptoms in pediatric heart transplant recipients. Health Psychology Research, 2(2), 63-66.

Falkenstein, K., Flynn, L., Kirkpatrick, B., Casa-Melley, A., \& Dunn, S. (2004). Non-compliance in children postliver transplant: Who are the culprits. Pediatric Transplantation, 8(3), 233-36.

Foster, B. J., Pai, A. L.H., Zelikovsky, N., Amaral, S., Bell, L., Dharnidharka, V. R., . . Furth, S. L. (2018). A randomized trial of multicomponent intervention to promote medication adherence: The teen adherence in kidney transplant effectiveness of intervention trial (TAKE-IT). American Journal of Kidney Disease, 72(1), 30-41.

Fredericks, E. M., \& Dore-Stites, D. (2011). Adherence to immunosuppressants: How can it be improved in adolescent organ transplant recipients. Curr Opin Organ Transplant, 15(5), 614-620.

Gilleland, J., Amaral, S., Mee, L., \& Blount, R. (2012). Getting ready to leave: Transition readiness in adolescent kidney transplant recipients. Journal of Pediatric Psychology, 37(1), 85-96.

Griffin, K. J., \& Elkin, T. D. (2001). Non-adherence in pediatric transplantation: A review of the existing literature. Journal of Pediatric Transplantation, 5(4), 246-249.

Gutierrez-Colina, A. M., Eaton, C. K., Lee, J. L., Reed-Knight, B., Loiselle, K., Mee, L. L., . . Blount, R. L. (2016). Executive functioning, barriers to adherence, and non adherence in adolescent and young adult transplant recipients. Journal of Pediatric Psychology, 41(7), 759-767.

Hanghøj, S., \& Boisen, K. A. (2014). Self-reported barriers to medication adherence among chronically ill adolescents: A systematic review. Journal of Adolescent Health, 54(2), 121-138.

Huang, J. S., Terrones, L., Tompane, T., Dillon, L., Plan, M., Gottschalk, M., . . Bartholomew, L. K. (2014). Preparing adolescents with chronic disease for transition to adult care: A technology program. Pediatrics, 133(6), 16391646.

Improving adolescent adherence rates. (2014, March 31). Retrieved May 6, 2019, from Children's Hospital of Philadelphia website: https://www.chop.edu

Johnson, S., Sidelinger, D. E., Blanco, E., Palinkas, L. A. P. A., Macdonald, D., \& Reznik, V. (2008). Ethnic differences and treatment trajectories in chronic kidney disease. Journal of Health Care for the Poor and Underserved, 19(1), 90-102. Retrieved from http://muse.jhu.edu

Kim, B., \& White, K. (2017). How can health professionals enhance interpersonal communication with adolescent and young adults to improve health care outcomes?: Systematic literature review. International Journal of Adolescence and Youth, 23(2), 198-218.

Kirklin, J. K. (2015). Current challenges in pediatric heart transplantation for congenital heart disease. Current Opinion in Organ Transplantation, 20(5), 577-583. 
Klostermann, B. K., Slap, G. B., Nebrig, D. M., Tivorsak, T. L., \& Britto, M. T. (2005). Earning trust and losing it: Adolescents' views on trusting physicians. The Journal of Family Practice, 54(8). Retrieved from https://www.mdedge.com

Lawrence, K., Stilley, C. S., Olshansky, E., Bender, A., \& Webber, S. A. (2008). Further exploration: Maturity and adherence in adolescent and young adult heart transplant recipients. Progress in Transplantation, 18(1), 50-54.

Lochridge, J., Wolff, J., Olivia, M., \& O'Sullivan-Oliveira, J. (2013). Perceptions of solid organ transplant recipients regarding self-care management and transitioning. Pediatric Nursing, 39(2), 81-89. Retrieved from http://eds.b.ebscohost.com

McCormick King, M. L., Mee, L. L., Gutierrez-Colina, A. M., Eaton, C. K., Lee, J. L., \& Blount, R. L. (2014). Emotional functioning, barriers, and medication adherence in pediatric transplantation recipients. Journal of Pediatric Psychology, 39(3), 283-293.

Miloh, T., Annunziato, R., Arnon, R., Warshaw, J., Parkar, S., Suchy, F. J., . . Kerkar, N. (2009). Improved adherence and outcomes for pediatric liver transplant recipients by using text messaging. Pediatrics, 124(5), 844-850.

Pruette, C. (2019, April 26). [Personal interview by the author].

Rianthavorn, P., Ettenger, R. B., Malekzadeh, M., Marik, J. L., \& Struber, M. (2004). Noncompliance with immunosuppressive medications in pediatric and adolescent patients receiving solid-organ transplants. Transplantation, 77(5), 778-782.

Sabaté, E. \& World Health Organization. (2001, June). Noncommunicable Diseases and Mental Health Cluster: Vol. 4-5. Adherence to long-term therapies: policy for action. Retrieved from http://www.who.int

Shaw, R. J., Palmer, L., Blasey, C., \& Sarwal, M. (2003). A typology of non-adherence in pediatric renal transplant recipients. Pediatric Transplantation, 7(6), 489-493.

Shellmer, D., Dabbs, A., \& Dew, M. (2011). Medical adherence in pediatric organ transplantation: What are the next steps? Current Opinion in Organ Transplantation., 16(5), 509-514.

Simons, L. E., McCormick, M. L., Devine, K., \& Blount, R. L. (2010). Medication barriers predict adolescent transplant recipients adherence and clinical outcomes at 18-month follow-up. Journal of Pediatric Psychology, 35(9), 1038-1048.

Simons, L. E., McCormick, M. L., Mee, L. L., \& Blount, R. L. (2009). Parent and patient perspectives on barriers to medication adherence in adolescent transplant recipients. Pediatric Transplantation, 13(3), 338-347.

Steuer, R., \& McCauley, S. O. (2017). Maintaining the Gift of Life: Achieving Adherence in Adolescent Heart Transplant Recipients. Journal of Pediatric Health Care, 31(5), 546-554.

Stuber, M. L. (2011). Psychiatric issues in pediatric organ transplantation. Pediatric Clinics of North America, $58(4), 285-300$. 
Taddeo, D., Egedy, M., \& Frappier, J.-Y. (2008). Adherence to treatment in adolescents. Pediatrics and Child Health, 13(1), 19-24. Retrieved from https://www.ncbi.nlm.nih.gov

Taylor, R. M., Franck, L. S., Dhawan, A., \& Gibson, F. (2010). The stories of young people living with a liver transplant. Qualitative Health Research, 20(8), 1076-1090.

Wray, J., Waters, S., Radley-Smith, R., \& Sensky, T. (2006). Adherence in adolescent and young adults following heart or lung transplantation. Pediatric Transplantation, 10(6), 694-700.

Zelikovsky, N., Schast, A. P., Palmer, J., \& Meyers, K. E.C. (2008). Perceived barriers to adherence among adolescent renal transplant candidates. Pediatric Transplantation, 12(3), 300-308. 